\title{
Hawking Radiation and Deflection of Light from Rindler Modified Schwarzschild Black Hole
}

\author{
I. SAKALli ${ }^{1}$ and A. Ovgun ${ }^{2,1}$ \\ 1 Physics Department, Eastern Mediterranean University, Famagusta, Northern Cyprus, \\ Turkey \\ 2 Instituto de Física, Pontificia Universidad Católica de Valparaíso, Casilla 4950, Val- \\ paraiso, Chile
}

PACS 04.20.Gz - Classical general relativity

PACS 04.70.Dy - Quantum aspects of black holes, evaporation, thermodynamics

PACS 02.40.Hw - Classical differential geometry

\begin{abstract}
We investigate the Hawking radiation of massive spin-1 vector particles, which are coupled to vacuum fluctuations of a quantum field, from Rindler modified Schwarzschild black hole. Rindler acceleration is used to produce the post-general relativistic theory of gravity for the distant field of a point mass. The gravitational lensing problem of the Rindler modified Schwarzschild black hole is also studied. We compute the deflection angle for the IR region (large distance limit as infrared) by using the Gaussian curvature of the optical metric of this back hole. Our investigations clarify how the Rindler acceleration plays a role on the Hawking radiation and gravitational lensing.
\end{abstract}

Introduction. - Until the mid-1970s, black holes (BHs) were believed to be such super absorbent objects in the universe that nothing can come out from them. In 1974, Hawking [1,2 showed that a $\mathrm{BH}$ can thermally create and emit virtual particles until it snuffs out its energy. This condition means that if a BH does not gain mass from any other source, then it could eventually shrink itself out of existence and evaporate completely; this process is called Hawking radiation (HR). HR is an intriguing puzzle that results from the amalgamation of general relativity and quantum mechanics. Hawking 1, 2, showed that black hole evaporation cannot be the result of unitary evolution of a pure state. Thus, the process of gravitational collapse is incompatible with the standard principles of quantum mechanics. In fact, the radiation emitted by a $\mathrm{BH}$ at late times is exactly described by a thermal density matrix (modulated by a greybody factor). A density matrix is a well-known matrix that describes a quantum system in a mixed state (a statistical ensemble of several quantum states). Therefore, as is the case with black-body radiation, HR quanta do not carry information. No mathematical transformation exists between the unitary operator and the density matrix. Thus, the information encoded in the wave function is irretrievably lost; this process is known as the so-called information loss paradox [3]. Efforts to resolve this problem have been continuing even today. A reader who wants to learn the details and recent developments about the information loss paradox may refer to $[4 \sqrt{6}$. On the other hand, studies 7,9 on the density matrix of a $\mathrm{BH}$ radiation have recently attracted much attention. It has been shown that small fluctuations of the $\mathrm{BH}$ horizon give rise to corrections to the density matrix of the HR. The associated corrections are shown to be
9

10 11 12 13 14 15 \section{1}


the correlations between the HR and the background geometry [9]. It is worth noting that correlations account for the information stored in the BH with the collapsing matter. Since the original studies of $\mathrm{BH}$ emission [1,2], works on the $\mathrm{HR}$ have been still continuing. To date, HR was verified by various methods for many different types of particles having spin $s=0,1 / 2,1,3 / 2,2, \ldots 10,24,89,92$. HR studies include the lower and higher dimensional BHs, wormholes, and black strings 25, 26, 29, 34. Recent studies 35 37] have claimed that HR has been observed in the laboratory environment. Those experiments about HR were conducted by Steinhauer, who used a sonic (or the so-called analogue) BH in an atomic Bose-Einstein condensate 38 so that a state, where gas bosons are cooled to temperatures very close to absolute zero (that is, very near $0 \mathrm{~K}$ or $-273.16^{\circ} \mathrm{C}$ ). Thus, the sonic $\mathrm{BH}$ could mimic a real cosmic event horizon. At this stage, Steinhauer managed to observe the particles of sound (phonons) at the BH's event horizon and found that sound waves in the Bose-Einstein condensate obey Hawking's theory 37. However, many physicists are still cautious about these results. Other experiments are needed to support the experiment of Steinhauer.

In particle physics, spin-1 particles are called vector particles. The most well-known massive spin-1 particles, which are described by a three-dimensional spin vector are the weak intermediate vector bosons $Z$ and $W^{ \pm}$, and the mesons $\boldsymbol{\Upsilon}, \mathbf{J} / \Psi, \boldsymbol{\phi}$, and $\boldsymbol{\rho}[39]$. Photons are the massless spin-1 particles, which can only be directed parallel or anti-parallel to their direction of motion. Free massive spin-1 fields are governed by the Proca field equation, and massless spin-1 fields are governed by the Maxwell field equation. Compelling evidence suggests that the spin-1 fields are potential dark matter candidates 4042$]$. In recent years, HR of the spin-1 particles have attracted considerable interest 4349 .

In this study, we consider the Rindler modified Schwarzchild BH (RMSBH) 50 51, which was initially proposed by Grumiller to explain the mysterious attractive constant radial force that acts on the Pioneer spacecrafts 52 . The force law obtained from Rindler's acceleration term has a considerable effect on the gravity at very long distances. However, Turyshev et al. 57. showed that the Pioneer anomaly is due to the thermal heat loss of the satellites. Nevertheless, RMSBH is still on the agenda because it provides a basis for the theoretical explanation of the following issues: rotation curves of spiral galaxies, gravitational redshift, and perihelion shift in planetary orbits. Previous studies of the RMSBH, which include subjects of spectroscopy of area/entropy, quantum tunneling, and geodesics, can be seen in 58 60. The Rindler acceleration is also used in a quantum gravity corrected gravity theories to explain the rotation of the curve formula for the local galaxies 50 52. The new Rindler acceleration term is studied as an alternative of the dark matter in galaxies and it is checked by using 8 galaxies of the HI Nearby Galaxy Survey which gives the Rindler acceleration parameter of around $a \approx 3 * 10^{-9} \mathrm{~cm} / \mathrm{s}^{2}[5356$.

Light is electromagnetic radiation within a certain portion of the electromagnetic spectrum. On the other hand, HR is mainly a thermal radiation. The main purpose of this paper is to study the two possible problems about the radiation physics (flow of atomic and subatomic particles and of waves, such as those that characterize heat rays, light rays, and X-rays) of the RMSBH: HR of the RMSBH and gravitational lensing of the RMSBH. To this end, we first study the HR of the massive spin-1 particles tunneling from the RMSBH. We apply the Hamilton-Jacobi and the complex path integration quantum tunneling methods 12, 13,61 to the Proca equation 433 and obtain a set of differential equations. Following 4347 , we set the determinant of coefficient matrix of the equation to zero to obtain a non-trivial solution. Thus, we obtain the leading order term of the classical action $\left(S_{0}\right)$ of the vector particles that are outgoing/ingoing from the horizon. We finally derive the tunneling rate of the spin-1 particles in the RMSBH and read the Hawking temperature of the RMSBH. The phenomenon of gravitational lensing, which was predicted by Einstein's theory of general relativity, is a side effect of light moving along the curvature of spacetime, where the light that passes near a massive object is deflected slightly toward the mass. This phenomenon was observed for the first time in 1919 by Eddington and Dyson during a solar 
eclipse [62]. Since then, gravitational lensing has been one of the important tools in astronomy and astrophysics. For more details and recent contributions about gravitational lensing, the reader may refer to $27,28,63,67$. We will also study the gravitational lensing problem of the RMSBH. For this purpose, we follow the geometrical method of Gibbons and Werner [68. In this manner, we explore the effect of the Rindler acceleration on the deflection of light moving in the IR region of the RMSBH. The paper is organized as follows: In Section II, we introduce the physical features of the RMSBH geometry. Section III is devoted to the computation of the HR of the massive spin- 1 particles from the RMSBH. In Section IV, we study the deflection of light from the RMSBH at the IR region via the method of Gibbons and Werner 68. Our results are summarized and discussed in Section V. (Throughout the paper, we use units in which fundamental constants are $G=c=k_{B}=1$ ).

RMSBH Geometry. - Grumiller [50] constructed an effective model for the gravity of a central object at large scale (i.e. outside the galaxy) called the RMSBH geometry. The Rindler term in the RMSBH spacetime causes an anomalous acceleration in the geodesics of test particles. RMSBH is the solution to the generic effective theory of gravity described by the following action:

$$
S=-\int d^{2} x \sqrt{-g}\left[\Phi^{2} R+2(\partial \Phi)^{2}+8 a \Phi-6 \Lambda \Phi^{2}+2\right]
$$

where $g=\operatorname{det}\left(g_{\mu \nu}\right)$ is the determinant of the metric tensor, $\Phi$ denotes the scalar field, $\Lambda$ is the cosmological constant, $R$ is the Ricci scalar and $a$ stands for the Rindler acceleration. The Rindler acceleration is for explaining about the Pioneer anomaly and also the shape of galactic rotation curve 50,52 . After applying the variational principle to action (1) and solving the corresponding field equations, one can obtain the following spherically symmetric line-element that models the IR gravity:

$$
d s^{2}=-f d t^{2}+\frac{1}{f} d r^{2}+r^{2}\left(d \theta^{2}+\sin ^{2} \theta d \phi^{2}\right),
$$

where

$$
f=1-\frac{2 M}{r}-\Lambda r^{2}+2 a r
$$

Metric (2) is nothing but the RMSBH spacetime. In Eq. (3), the quantity $M$ is an integral constant. When $a=\Lambda=0$, one easily recovers the Schwarzschild solution, with $M$ being the BH mass. Moreover, if $M=\Lambda=0$, then line-element (2) is the two-dimensional Rindler metric [69]. The value of $\Lambda$ is approximated to $10^{-123}$ [70,71]; therefore, we set it to zero $(\Lambda=0)$ for simplicity. Moreover, at the IR region, the value of the Rindler acceleration is estimated as $a \approx 10^{-62}-10^{-61}[50$. Metric function (3) can be rewritten as

$$
f=\frac{2 a}{r}\left(r-r_{h}\right)\left(r-r_{n}\right),
$$

in which

$$
r_{n}=-\frac{1+\sqrt{1+16 a M}}{4 a} .
$$

Since the radial coordinate $r$ is defined in the range of $0 \leq r<\infty$, it is therefore clear that $r_{n}$ (having negative value) is not a physical quantity, and thus it cannot be interpreted as the horizon (see for example 72]). So, RMSBH spacetime possesses only one horizon, which is called the event horizon $\left(r_{h}\right)$ :

$$
r_{h}=\frac{-1+\sqrt{1+16 a M}}{4 a} .
$$

The Bekenstein-Hawking entropy 69,73 of the RMSBH is given by

$$
S_{B H}=\frac{A_{h}}{4 \hbar}=\frac{\pi r_{h}^{2}}{\hbar}
$$


where $A_{h}=4 \pi r_{h}^{2}$ is the surface area of the RMSBH. The following conventional definition of the surface gravity defined for a spherically static spacetime 73$]$ is used:

$$
\kappa=\left.\frac{\partial_{r} f}{2}\right|_{r=r_{h}}=a\left(1-\frac{r_{n}}{r_{h}}\right),
$$

where a prime on a function denotes differentiation with respect to $r$. From here on, we read the Hawking temperature of the RMSBH as follows:

$$
T_{H}=\frac{\hbar \kappa}{2 \pi}=\frac{a \hbar}{2 \pi}\left(1-\frac{r_{n}}{r_{h}}\right) .
$$

Figure 1 shows how the Hawking temperature changes with Rindler acceleration in the RMSBH geometry.

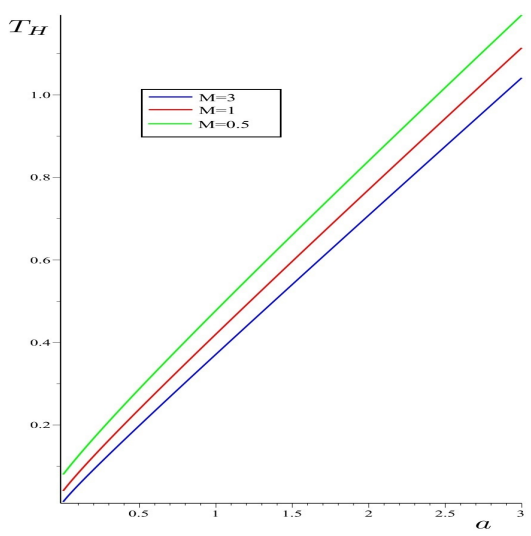

Fig. 1: Plots of $T_{H}$ versus $a$. The plots are governed by Eq. (9). Each color represents a RMSBH with different mass.

Quantum Tunneling of Massive Vector Particles from RMSBH. - In this section, we compute the HR of massive vector particles, which quantum mechanically tunnel from the RMSBH. We consider the Proca equation [43]:

$$
\frac{1}{\sqrt{-g}} \partial_{\mu}\left(\sqrt{-g} \Psi^{\nu \mu}\right)+\frac{m^{2}}{\hbar^{2}} \Psi^{\nu}=0,
$$

which corresponds to the wave equation of a spin-1 field $\Psi_{\nu}$ of mass $m$. In Eq. (10), the second rank tensor is defined by

$$
\Psi_{\mu \nu}=\partial_{\mu} \Psi_{\nu}-\partial_{\nu} \Psi_{\mu} .
$$

Considering the WKB method, ansatz of the spin-1 field can be defined by [44]|74

$$
\Psi_{\nu}=C_{\nu} \exp \left(\frac{i}{\hbar}\left(S_{0}(t, r, \theta, \phi)+\hbar S_{1}(t, r, \theta, \phi)+\ldots\right)\right),
$$

where $S_{0}(t, r, \theta, \phi)$ is the kinetic term equal to the classical action of the particles 24, $S_{j=1,2, . .}(t, r, \theta, \phi)$ are the higher order action corrections, and $C_{\nu}=\left(C_{1}, C_{2}, C_{3}, C_{4}\right)$ represents some arbitrary constants. Furthermore, taking cognizance of the Hamilton-Jacobi method which takes the advantage of the symmetry (Killing vectors) of the spacetime 74, one can set the leading order of the action to 75

$$
S_{0}(t, r, \theta, \phi)=-E t+R(r, \theta)+j \phi+k,
$$


where $E$ and $j$ denote the energy and angular momentum of the vector particles, respectively. $k$ is a complex constant.

After manipulating the Proca equation (10) with Eq.s (12) and (13), we obtain a quadruple equation set to the lowest order in $\hbar$ as follows

$$
\begin{aligned}
& -E\left(\partial_{r} R\right) C_{1}-\frac{E\left(\partial_{\theta} R\right) C_{2}}{r^{2} f}-\frac{\left[\sin ^{2} \theta\left(r^{2} f\left(\partial_{r} R\right)^{2}+m^{2} r^{2}+\left(\partial_{\theta} R\right)^{2}\right)+j^{2}\right] C_{4}}{r^{2} f \sin ^{2} \theta} \\
& -\frac{E j C_{3}}{r^{2} f \sin ^{2} \theta}=0 \\
& -\frac{\left[-\sin ^{2} \theta f\left(\partial_{\theta} R\right)^{2}-f\left(m^{2} r^{2} \sin ^{2} \theta+j^{2}\right)+E^{2} r^{2} \sin ^{2} \theta\right] C_{1}}{r^{2} \sin ^{2} \theta}-\frac{f\left(\partial_{r} R\right)\left(\partial_{\theta} R\right) C_{2}}{r^{2}} \\
& -\frac{j f\left(\partial_{r} R\right) C_{3}}{r^{2} \sin \theta}-E\left(\partial_{r} R\right) C_{4}=0, \\
& -\frac{f\left(\partial_{r} R\right)\left(\partial_{\theta} R\right) C_{1}}{r^{2}}-\frac{\left[-\sin ^{2} \theta f\left(\partial_{r} R\right)^{2}-f\left(m^{2} r^{2} \sin ^{2} \theta+j^{2}\right)+E^{2} r^{2} \sin ^{2} \theta\right] C_{2}}{r^{2} \sin ^{2} \theta} \\
& -\frac{j\left(\partial_{\theta} R\right) C_{3}}{r^{4} \sin ^{2} \theta}-\frac{\left(\partial_{\theta} R\right) E C_{4}}{r^{2} f}=0, \\
& -\frac{j f\left(\partial_{r} R\right) C_{1}}{r^{2} \sin ^{2} \theta}-\frac{j\left(\partial_{\theta} R\right) C_{2}}{r^{4} \sin ^{2} \theta}+\frac{\left[f\left(\partial_{\theta} R\right)^{2}-r^{2}\left(-f^{2}\left(\partial_{r} R\right)^{2}-m^{2} f+E\right)\right] C_{3}}{r^{4} f \sin ^{2} \theta} \\
& -\frac{j E C_{4}}{r^{2} \sin ^{2} \theta f}=0
\end{aligned}
$$

After this stage, we can obtain a $4 \times 4$ coefficient matrix of $\aleph\left(C_{1}, C_{2}, C_{3}, C_{4}\right)^{T}=0$ in which the superscript $T$ represents the transition to the transposed vector. The non-zero components of $\aleph$ matrix are given by

$$
\begin{aligned}
& \aleph_{11}=\aleph_{24}=-E\left(\partial_{r} R\right), \\
& \aleph_{12}=\aleph_{34}=-\frac{E\left(\partial_{\theta} R\right)}{r^{2} f}, \\
& \aleph_{13}=\aleph_{44}=-\frac{E j}{r^{2} f \sin ^{2} \theta}, \\
& \aleph_{14}=-\frac{\left[\sin ^{2} \theta\left(r^{2} f\left(\partial_{r} R\right)^{2}+m^{2} r^{2}+\left(\partial_{\theta} R\right)^{2}\right)+j^{2}\right]}{r^{2} f \sin ^{2} \theta}, \\
& \aleph_{21}=-\frac{\left[-\sin ^{2} \theta f\left(\partial_{\theta} R\right)^{2}-f\left(m^{2} r^{2} \sin ^{2} \theta+j^{2}\right)+E^{2} r^{2} \sin ^{2} \theta\right]}{r^{2} \sin ^{2} \theta}, \\
& \aleph_{22}=\aleph_{31}=-\frac{f\left(\partial_{r} R\right)\left(\partial_{\theta} R\right)}{r^{2}},
\end{aligned}
$$




$$
\begin{aligned}
& \aleph_{23}=\aleph_{41}=-\frac{j f\left(\partial_{r} R\right)}{r^{2} \sin \theta}, \\
& \aleph_{32}=-\frac{\left[-\sin ^{2} \theta f\left(\partial_{r} R\right)^{2}-f\left(m^{2} r^{2} \sin ^{2} \theta+j^{2}\right)+E^{2} r^{2} \sin ^{2} \theta\right]}{r^{2} \sin ^{2} \theta}, \\
& \aleph_{33}=\aleph_{42}=-\frac{j\left(\partial_{\theta} R\right)}{r^{4} \sin ^{2} \theta}, \\
& \aleph_{43}=\frac{\left[f\left(\partial_{\theta} R\right)^{2}-r^{2}\left(-f^{2}\left(\partial_{r} R\right)^{2}-m^{2} f+E\right)\right]}{r^{4} f \sin ^{2} \theta} .
\end{aligned}
$$

The non-trivial solution of the quadruple equation set is conditional on $\operatorname{det} \aleph=0$. Thus, we have

$$
\frac{m^{2}\left[\sin ^{2} \theta f\left(\partial_{\theta} R\right)^{2}+f^{2} r^{2}\left(\partial_{r} R\right)^{2}+f\left(m^{2} \sin ^{2} \theta r^{2}+j^{2}\right)-E^{2} r^{2} \sin \theta\right]^{3}}{r^{10} \sin \theta f^{3}}=0,
$$

which yields the integral solution of the radial function as

$$
R_{ \pm}=\int \pm \frac{\sqrt{E^{2}-f\left(m^{2}+\frac{\left(\partial_{\theta} R\right)^{2}}{r^{2}}+\frac{j^{2}}{\sin ^{2} \theta r^{2}}\right)}}{f} .
$$

$R_{+}(r)$ and $R_{-}(r)$ correspond to the outgoing (i.e., emission) and ingoing (i.e., absorption) vector particles from/to the RMSBH, respectively. Because of a pole located at the horizon, the imaginary part of $R_{ \pm}(r)$ can be calculated by using the complex path integration method [12.13. Therefore, we can evaluate the integral (29) in the vicinity of the horizon as

$$
\operatorname{Im} W_{ \pm}(r)= \pm\left.\frac{\pi}{\partial_{r} f} E\right|_{r=r_{h}} .
$$

The probabilities of the massive vector particles, tunneling from the horizon of RMSBH, become

$$
\begin{aligned}
P_{\text {emission }} & =e^{-\frac{2}{\hbar} \operatorname{Im} S_{+}}=e^{\left[-\frac{2}{\hbar}\left(\operatorname{Im} W_{+}+\operatorname{Im} \mathbb{k}\right)\right]}, \\
P_{\text {absorption }} & =e^{-\frac{2}{\hbar} \operatorname{Im} S_{-}}=e^{\left[-\frac{2}{\hbar}\left(\operatorname{Im} W_{-}+\operatorname{Im} \mathbb{k}\right)\right]} .
\end{aligned}
$$

According to the classical concept of $\mathrm{BH}$ physics, the ingoing vector particles must be fully absorbed, as indicated by $P_{\text {absorption }}=1$. This requirement can be fulfilled with $I m \mathbb{k}=-I m W_{-}$. Recalling $W_{+}=-W_{-}$, we can thus compute the quantum tunneling rate of the massive vector particles of the RMSBH as

$$
\begin{array}{r}
\Gamma=P_{\text {emission }}=\exp \left(-\frac{4}{\hbar} \operatorname{Im} W_{+}\right) \\
=\exp \left(-\left.\frac{4 \pi}{\hbar\left(\partial_{r} f\right)} E\right|_{r=r_{h}}\right) .
\end{array}
$$

$\Gamma$ is also equivalent to the Boltzmann factor 24 . The latter remark enables us to compute the surface temperature of the RMSBH as follows:

$$
T=\left.\frac{\hbar\left(\partial_{r} f\right)}{4 \pi}\right|_{r=r_{h}}=\frac{a \hbar\left(r_{h}-r_{n}\right)}{2 \pi r_{h}} .
$$

The above result completely overlaps with the Hawking temperature (9) of the RMSBH. The Hawking temperature of the RMSBH increases proportionally with the Rindler acceleration, which can be also best seen in Fig. (1). 
Deflection of Light from RMSBH. - In this section, we will study the deflection of null geodesics from the RMSBH, that is, the gravitational lensing problem. The main reason to combine the Hawking radiation and the deflection of light is that the gravitational lensing is a observational effect to help to model the theoretical theories, made new claim that due to the interaction of the galaxies, the normal matter is separated from the dark matter after collision of galaxies then the bending of light is occurred due to the the dark matter from the cluster differently to the case without dark matter. Moreover, increasing number of new observations give evidences to propose that the unknown particles of dark matter may have another property that is that they can make self-interaction, namely an exchange of dark photons which have spin-1 may create the force. In this work, by investigating radiation of spin-1 particles, such as dark photons from the black hole, we find the evidence of dark matter. Moreover, astronomers may have an extremely powerful tool to seeing the gravitational effects of the dark matter using the gravitational lensing. On this purpose, weak lensing occurs when the light from a distant galaxy passes a distance from a darkmatter concentration and produces a slight distortion in the shape of a distant galaxy.

To this end, we will suppose that RMSBH consists of a perfect fluid, which can be thought of as the stellar fluid of a cluster of galaxies. Such stellar fluids act as a gravitational lens, which is capable of bending the light from the source as the light travels toward the observer. By using the static and spherically symmetric feature of metric (2), without loss of generality, we can assume that null geodesics $\left(d s^{2}=0\right)$ lie in the equatorial plane: $\theta=\pi / 2$. Therefore, all images are collinear with the lens center $[76$. Light rays are the spatial projection of the null geodesics of the line element of the optical metric 77], which is given by

$$
d t^{2}=g_{i j}^{o m} d x^{i} d x^{j}
$$

where $g_{i j}^{o m}$ originates from Fermat's principle 78,79 :

$$
g_{i j}^{o m}=\frac{g_{i j}}{-g_{00}}
$$

Thus, metric (35) becomes

$$
d t^{2}=d r^{* 2}+F\left(r^{*}\right)^{2} d \phi^{2}
$$

where $r^{*}$ is the radial Regge-Wheeler tortoise coordinate

$$
d r^{*}=\frac{d r}{f}
$$

and

$$
F\left(r^{*}\right)=\frac{r}{\sqrt{f}}, \quad r=r\left(r^{*}\right) .
$$

We immediately deduce from Eq. (37) that the optical metric is a surface of revolution. The intrinsic or the so-called Gaussian curvature $\mathcal{K}$ [68] of the optical metric can be expressed as follows:

$$
\begin{aligned}
\mathcal{K} & =-\frac{1}{F\left(r^{*}\right)} \frac{d^{2} F\left(r^{*}\right)}{d r^{* 2}} \\
& =-\frac{1}{F\left(r^{*}\right)}\left[\frac{d r}{d r^{*}} \frac{d}{d r}\left(\frac{d r}{d r^{*}}\right) \frac{d F\left(r^{*}\right)}{d r}+\left(\frac{d r}{d r^{*}}\right)^{2} \frac{d^{2} F\left(r^{*}\right)}{d r^{2}}\right]
\end{aligned}
$$

The area element of the optical metric (35) is given by

$$
d A=\sqrt{\left|\operatorname{det} g^{0 m}\right|} d r d \phi=\frac{r}{\sqrt{f^{3}}} d r d \phi .
$$


We now consider a lens model of perfect fluid, which is characterized by the following energy momentum tensor [80]:

$$
T^{\alpha \beta}=\operatorname{diag}[\rho(r), p(r), p(r), p(r)],
$$

where $p(r)$ and $\rho(r)$ denote the pressure and density, respectively. If we set 80

$$
f=\frac{r-2 \mu(r)}{r},
$$

where

$$
\mu(r)=4 \pi \int_{0}^{r} \rho\left(r^{\prime}\right) r^{\prime 2} d r^{\prime},
$$

from Eq.s (3), (43), and (44), we obtain

$$
\int_{0}^{r} \rho\left(r^{\prime}\right) r^{\prime 2} d r^{\prime}=\frac{1}{4 \pi}\left(M-a r^{2}\right) .
$$

Therefore, the Tolman-Oppenheimer-Volkoff equation 80, 81, which ensues from the conservation of the energy momentum tensor $\left(\nabla_{\alpha} T^{\alpha \beta}=0\right)$, reads 80

$$
\frac{d p(r)}{d r}=-\frac{[\rho(r)+p(r)]\left[\mu(r)+4 \pi r^{3} p(r)\right]}{r^{2} f} .
$$

Equation (46), in fact, shows the hydrodynamical behavior of a massive compact astrophysical object in terms of mass, pressure, and energy density [80. We also have

$$
\frac{d f}{d r}=\frac{2}{r^{2}}\left[\mu(r)+4 \pi r^{3} p(r)\right] .
$$

By inserting Eq.s (46) and (47) into Eq. (40), we can obtain the Gaussian optical curvature as follows:

$$
\mathcal{K}=\frac{-2 \mu(r)}{r^{3}}\left\{f+\frac{\mu}{2 r}-\frac{4 \pi r^{3}}{\mu}\left[\rho(r) f+p(r)\left(f-\frac{\mu(r)}{r}-2 \pi p(r) r^{2}\right)\right]\right\} .
$$

Our main motivation is to study the weak gravitational lensing phenomenon, which is more realistic in the astrophysical observation. For this reason, we shall focus on the non-relativistic kinetic theory (e.g., the collisionless Boltzmann equation 82 of the nonrelativistic stellar fluids). This condition means that the pressure is ignored in Eq. (48) (see also [80]). Thus, the multiplication of the Gaussian optical curvature with the area element of the optic metric results in

$$
\mathcal{K} d A=\frac{-2 \mu(r)}{r^{2} \sqrt{f^{3}}}\left\{f+\frac{\mu(r)}{2 r}-\frac{4 \pi r^{3}}{\mu(r)}[\rho(r) f]\right\} d r d \phi .
$$

In the spirit of [68, RMSBH lens can be characterized by [see Eq. (45)]

$$
\rho(r) \approx-\frac{a}{2 \pi r} .
$$

However, in the weak field limit [corresponding to $r>2 M$ (see for example 83, 84)], similar to the Schwarzschild lens [68], the density vanishes. Therefore, Eq. (44) admits

$$
\mu(r) \approx \text { constant }
$$


and Eq. (49) becomes

$$
\mathcal{K} d A \approx \frac{-2 \mu(r)}{r^{2} \sqrt{f^{3}}}\left[f+\frac{\mu(r)}{2 r}\right] d r d \phi .
$$

The deflection angle $(\delta)$ is calculated by integrating the Gaussian curvature on the circular boundary of domain $D_{2}$ [68, which can be best seen from figure 1 of [68]. In the weak deflection limit, it is assumed that the light rays are governed by the function (at zeroth order) of

$$
r(t)=\frac{b}{\sin \phi}
$$

where $b$ is known as the impact parameter with $b \gg M$ 80. Furthermore, since we take cognizance of the IR region, Eq. (52) can be recast into the following expression by using the Taylor series

$$
\mathcal{K} d A \approx-\frac{1}{2 \sqrt{2}}\left(a r^{3}\right)^{-\frac{3}{2}} d r d \phi .
$$

Thus, $\delta$ is computed via the following integral 68

$$
\begin{aligned}
\delta & =-\iint_{D_{2}} \mathcal{K} d A, \\
& \approx \frac{1}{2 \sqrt{2}} \int_{0}^{\pi} \int_{b / \sin \phi}^{\infty}\left(a r^{3}\right)^{-\frac{3}{2}} d r d \phi, \\
& =\frac{10}{147 \sqrt{a^{3} b^{7}}} \text { EllipticK }\left(\frac{1}{\sqrt{2}}\right), \\
& \simeq \frac{0.126127529}{\sqrt{a^{3} b^{7}}} .
\end{aligned}
$$

The above result shows the RMSBH's gravitational lensing deflection angle at the IR region. Evidently, the deflection angle is inversely proportional to the Rindler acceleration, that is, gravitational lensing of an accelerated RMSBH is less than the almost non-accelerated RMSBH. The latter remark implies that observing (via the gravitational lensing) RMSBH will be more difficult than observing Schwarzschild BH. Meanwhile, we should not overlook the point that $a^{3} b^{7}$ would have a significant value because the impact parameter $b \gg \mu$ in the denominator of Eq. (55). This finding means that the deflection angle possesses a small value at the IR region.

Conclusion. - In this paper, two problems of radiation physics about the RMSBH were considered. We focused on the HR of massive spin-1 particles and gravitational lensing of the RMSBH, respectively. In fact, massive spin-1 particles are a potential dark matter candidate 40 and one of the most successful techniques to explore the dark matter has so far been the effect of gravitational lensing 85]. Although our work is purely theoretical, we believe that such theoretical studies will lead to the observation of the dark matter in future. To investigate the HR of massive vector particles, we employed the Proca equation (10) in the RMSBH background within the concept of semiclassical WKB approximation. By taking into account the Hamilton-Jacobi and the complex path integration methods [12,13, we computed the tunneling rate (28) of the RMSBH, which is ruled by the wellknown Boltzmann factor. Then, the temperature obtained from the resulting tunneling rate is shown to be same as the original Hawking temperature (9) of the RMSBH. The Hawking temperature of the RMSBH increases proportionally with the Rindler acceleration, which can be best seen in Fig. (1). We also applied the geometrical approach of the gravitational lensing theory to the RMSBH. The optical metric (37) in which the geodesics are the spatial light rays of the RMSBH was derived to calculate the Gaussian curvature in the weak deflection limit. At the IR region, deflection angle (54) decreases with the increasing Rindler acceleration value, that is, the gravitational lensing of the RMSBH is always less than the 
Sakalli et al.

Schwarzschild BH. The gravitational lensing of a rotating RMSBH, which can be easily obtained by the Newman-Janis algorithm 86, 87, would be interesting to explore. In a rotating geometry, images are no longer collinear with the lens center 88. Therefore, such a problem may reveal more information compared with the present case. This issue is the next stage of study that interests us.

$$
* * *
$$

This work was supported by the Chilean FONDECYT Grant No. 3170035 (AÖ). We thank the Editor and anonymous Referee for their constructive comments and suggestions, which helped us improve the manuscript.

\section{REFERENCES}

[1] S. W. Hawking, Nature (London) 248, 30 (1974).

[2] S. W. Hawking, Commun. Math. Phys. 43, 199 (1975); 46, 206 (1976), Erratum.

[3] V. Balasubramanian and B. Czech, Class. Quantum Grav. 28, 163001 (2011).

[4] Y. C. Ong, Evolution of Black Holes in Anti-de Sitter Spacetime and the Firewall Controversy (Springer, New York, 2016)

[5] S. W. Hawking, M. J. Perry, and A. Strominger, Phys. Rev. Lett. 116, 231301 (2016).

[6] I. Sakalli and A. Ovgun, EPL 110, 10008 (2015).

[7] R. Brustein and A. J. M. Medved, J. High Energy Phys. 09, 015 (2013).

[8] R. Brustein and A. J. M. Medved, J. High Energy Phys. 02, 116 (2014).

[9] M. Z. Iofa, Phys. Rev D 94, 064044 (2016).

[10] M. K. Parikh and F. Wilczek, Phys. Rev. Lett. 85, 5042 (2000).

[11] M. K. Parikh, Int. J. Mod. Phys. D 13, 2351 (2004).

[12] M. Angheben, M. Nadalini, L. Vanzo and S. Zerbini, J. High Energy Phys. 05, 014 (2005).

13] M. Angheben, M. Nadalini, L. Vanzo, and S. Zerbini, J. High Energy Phys. 05, 037 (2005).

[14] K. Srinivasan and T. Padmanabhan, Phys. Rev. D 60, 024007 (1999).

[15] E. T. Akhmedov, V. Akhmedova, and D. Singleton, Phys. Lett. B 642, 124 (2006).

[16] E. T. Akhmedov, V. Akhmedova, T. Pilling, and D. Singleton, Int. J. Mod. Phys. A 22, 1705 (2007).

[17] V. Akhmedova, T. Pilling, A. de Gill and D. Singleton, Phys. Lett. B 666, 269 (2008).

[18] B. R. Majhi, Phys. Rev. D 79, 044005, 2009.

[19] H. Pasaoglu and I. Sakalli, Int. J. Theor. Phys. 48, 3517 (2009).

[20] I. Sakalli, M. Halilsoy, and H. Pasaoglu, Int. J. Theor. Phys. 50, 3212 (2011).

[21] A. Övgün, Int.J.Theor.Phys. 55 no.6, 2919-2927 (2016).

[22] I. Sakalli, M. Halilsoy, H. Pasaoglu, Astrophys. Space Sci. 340, 155 (2012); 357, 95 (2015), Erratum.

[23] I. Sakalli and S. F. Mirekhtiary, J. Exp. Theor. Phys. 117, 656 (2013).

[24] L. Vanzo, G. Acquaviva and R. Di Criscienzo, Class. Quantum Gravity 28, 18 (2011).

[25] I. Sakalli and A. Ovgun, Astrophys. Space Sci. 359, 32 (2015).

[26] I. Sakalli, A. Övgün, K. Jusufi, Astrophys.Space Sci. 361 no.10, 330 (2016).

[27] K. Jusufi, I. Sakalli and A. Övgün, arXiv:1705.06197.

[28] K. Jusufi, M. C. Werner, A. Banerjee, A. Ovgun, Phys.Rev. D 95, no.10, 104012 (2017).

[29] R. da Rocha and J. M. Hoff da Silva, EPL 107, 50001 (2014).

[30] H. Gursel and I. Sakalli, Can. J. Phys. 94, 147 (2016).

[31] J. Ahmed and K. Saifullah, JCAP 08, 011 (2011).

[32] I. Sakalli and H. Gursel, Eur. Phys. J. C 76, 318 (2016).

[33] T. Pappas, P. Kanti, and N. Pappas, Phys. Rev. D 94, 024035 (2016).

[34] X. Calmet, Quantum Aspects of Black Holes (Fundamental Theories of Physics) (Springer, New York, 2015).

[35] J. Steinhauer, Nature Phys. 10, 864 (2014).

[36] J. Steinhauer, Phys. Rev. D 92, 024043 (2015).

[37] J. Steinhauer, Nature Phys. 12, 959 (2016).

[38] J. Macher and R. Parentani, Phys. Rev. A 80, 043601 (2009). 
[39] W. Greiner, Field Quantization (Springer-Verlag, Berlin, 1996).

[40] E. Gabrielli, L. Marzola, M. Raidal, and H. Veermäe, JHEP 08, 150 (2015).

[41] Jonathan L. Feng, et al., arXiv:1608.03591 (2016).

[42] Y. Kahn and J. Thaler, Phys. Rev. D 86, 115012 (2012).

[43] S. I. Kruglov, Mod. Phys. Lett. A 29, 1450203 (2014).

[44] S. I. Kruglov, Int. J. Mod. Phys. A 29, 1450118 (2014).

[45] I. Sakalli and A. Ovgun, J. Exp. Theor. Phys. 121, 404 (2015).

[46] I. Sakalli and A. Ovgun, Eur. Phys. J. Plus 130, 110 (2015).

[47] I. Sakalli and A. Ovgun, Gen. Relativ. Gravit. 48, 1 (2016).

[48] A. Ovgün and K. Jusufi, Eur.Phys.J.Plus 131, 177 (2016).

[49] K. Jusufi and A. Ovgun, Astrophys. Space Sci. 361, 207 (2016).

[50] D. Grumiller, Phys. Rev. Lett. 105, 211303 (2010).

[51] D. Grumiller, Phys. Rev. Lett. 106, 039901 (2011).

[52] D. Grumiller and F. Preis, Int. J. Mod. Phys. D 20, 2761 (2011).

[53] H. N. Lin, M. H. Li, X. Li, Z. Chang, MNRAS 430, 450 (2013).

[54] F. Walter, et al., Astron. J. 136, 2563 (2008).

[55] J. Mastache, J.L. Cervantes-Cota, A. de la Macorra, Phys. Rev. D 87, 063001 (2013).

[56] J. L. Cervantes-Cota and J. A. Gomez-Lopez, Physics Letters B 728, 537 (2014).

[57] S. G. Turyshev et al.,Phys. Rev. Lett. 108, 241101 (2012)

[58] S. F. Mirekhtiary and I. Sakalli, Commun. Theor. Phys. 61, 558 (2014).

[59] I. Sakalli and S. F. Mirekhtiary, Astrophys. Space Sci. 350, 727 (2014).

[60] M. Halilsoy, O. Gurtug, and S. H. Mazharimousavi, Gen. Relativ. Gravit. 45, 2363 (2013).

[61] S. Shankaranarayanan, K. Srinivasan, and T. Padmanabhan, Mod. Phys. Lett. A 16, 571 (2001).

[62] D. Kennefick, Physics Today 62, 37 (2009).

[63] M. Bartelmann and P. Schneider, Phys. Rep. 340, 291 (2001).

[64] M. Bartelmann, Class. Quantum Grav. 27, 233001 (2010).

[65] G. S. Bisnovatyi-Kogan and O. Yu. Tsupko, Gravit. Cosmol. 14, 226 (2008).

[66] K. Jusufi, Astrophys. Space Sci. 361, 24, (2016).

[67] X. Liu, N. Yang, and J. Jia, Class. Quantum Grav. 33, 175014 (2016).

[68] G. W. Gibbons and M. C. Werner, Class. Quantum Grav. 25, 235009 (2008).

[69] V. P. Frolov and I. D. Novikov, Black Hole Physics: Basic Concepts and New Developments (Kluwer Academic Publishers, London, 1998).

[70] S. Perlmutter et al., Astrophys. J. 517, 565 (1999).

[71] A. G. Riess et al., Astron. J. 116, 1009 (1998).

[72] M. Cataldo and A. Garcia, Phys. Rev. D 61, 084003 (2000).

[73] R. M. Wald, General Relativity (The University of Chicago Press, Chicago, 1984).

[74] R. Banerjee and B. R. Majhi, J. High Energy Phys. 06, 095 (2008).

[75] G. R. Chen, S. Zhou, and Y. C. Huang, Astrophys. Space Sci. 357, 51 (2015).

[76] S. Capozziello and G. Iovane, Phys. Lett. A 259, 185 (1999).

[77] S. Casey, Class. Quantum Grav. 29, 237001 (2012).

[78] C. Duval, Z. Horvathy, and P. A. Horvathy, Phys. Rev. D 74, 021701 (2006).

[79] I. Sakalli, A. Ovgun, and S. F. Mirekhtiary, Int. J. Geom. Methods Mod. Phys. 11, 1450074 (2014).

[80] N. Straumann, General Relativity: With Applications to Astrophysics (Springer, Berlin, 2004).

[81] K. Glampedakis, G. Pappas, H. O. Silva, and E. Berti, Phys. Rev. D 92, 024056 (2015).

[82] J. Binney and S. Tremaine, Galactic Dynamics (NJ: Princeton University Press, Princeton, 1987).

[83] S. Mendoza, T. Bernal, X. Hernandez, J. C. Hidalgo, and L. A. Torres, MNRAS 433, 1802 (2013).

[84] M. C. Campigotto, A. Diaferio, X.Hernandez, and L. Fatibene, arXiv:1612.01535 (2016).

[85] R. Massey, T. Kitching, and J. Richard, Rep. Prog. Phys. 73, 086901 (2010).

[86] A. J. Keane, Class. Quantum Grav. 31, 155003 (2014).

[87] H. Erbin, Gen. Relativ. Gravit. 47, 19 (2015).

[88] K. Beckwith and C. Done, Mon. Not. Roy. Astron. Soc. 359, 1217 (2005).

[89] R. Kerner, R.B. Mann, Phys. Rev. D 73, 104010 (2006).

[90] R. Kerner and R.B. Mann, Class. Quant. Grav. 25, 095014 (2008). 
Sakalli et al.

260 [91] R. Kerner and R.B. Mann, Phys. Lett. B 665, 277-283 (2008).

261 [92] Alexandre Yale, Robert B. Mann Phys. Lett. B 673, 168-172, (2009). 